(C2007 IEEE. Personal use of this material is permitted. However, permission to reprint/republish this material for advertising or promotional purposes or for creating new collective works for resale or redistribution to servers or lists, or to reuse any copyrighted component of this work in other works must be obtained from the IEEE. 


\title{
An investigation of factors affecting technology acceptance and use decisions by Australian allied health therapists
}

\author{
Louise K. Schaper, Graham P. Pervan \\ School of Information Systems, Curtin University of Technology, Perth Western Australia \\ l.schaper@curtin.edu.au; Graham.Pervan@cbs.curtin.edu.au
}

\begin{abstract}
The research reported in this paper describes the development, empirical validation and analysis of a model of technology acceptance by Australian occupational therapists. The study described involved the collection of quantitative data through a national survey, with over 2000 responses and a longitudinal case study within the community health sector. Results provide qualitative and quantitative support for the proposed model and demonstrate the inadequacy of traditional models of technology acceptance when applied to the health sector. This work extends technology acceptance studies into new realms of the health sector and highlights the need for a broadening of health IT research, and particularly technology acceptance studies, to encompass a more holistic and inclusive view of those who work in healthcare in order to gain a greater understanding of its complexities and how IS implementation success can be enhanced in this arena.
\end{abstract}

\section{Introduction}

In their editorial for this minitrack at HICSS 2004, Schuring and Spil stated "Developing, implementing and using information technology in organisations is a complex social activity. It is often characterised by ill-defined problems or vague goals, conflicts and disruptions that result from organisational change. Successfully implementing information systems in healthcare organisations appears to be a difficult task." [1]. In the three years that have passed since this inaugural minitrack, research on implementation, adoption and diffusion of information systems (IS) in healthcare has grown substantially.

A review of this literature shows that a common factor in this field of study is the inherent complexities involved in IS implementation in health [2], as well as a resistance by health professionals to accept and utilise information and communication technologies (ICT). This is a view supported by researchers and health informaticians alike and although the gap has decreased, the traditional lag in technology adoption in health which has been widely reported for over a decade [3-8] still holds true today - despite advances in technology, the increasingly high profile attributed to health ICT [9] and the potential benefits of adoption [10].

Another commonality in this research is the subject of enquiry. While the technologies studied range from telehealth [11], to clinical decision support tools [12], to electronic health records [13], to handheld computers [14] (amongst others); there is an almost exclusive focus on the acute care sector and the physicians who work within it [15-23]. Outside of the acute care sector, medical practitioners and their local practices are again the focus [24]. This focus on the medical profession is understandable, given the vital and omnipresent role they occupy within the sector. While this research is important and needs to continue, there is a concurrent need to ensure technology acceptance research is inclusive of all health professions across sectors and settings within health. The healthcare workforce is made up of a range of professionals including physicians, nurses, physiotherapists, occupational therapists, psychologists, dentists etc. all of whom play a unique and important role. While it could be stated that the goal of all health care professionals is similar - to improve the health and wellbeing of health consumers, each profession has a unique approach to achieving that goal and a unique set of skills, knowledge and frameworks that guide their practice. Following this logic, we can not presume to understand technology acceptance within health unless we study all the players in the system.

This marries recent calls for health-ICT agendas, strategies and research to broaden its focus to include long-term and chronic care [25, 26]. When considering technologies such as electronic health records the inclusion of chronic care seems obvious, as it is consumers with chronic health complaints that spend more time in the health sector and come into contact with a wider range of health professionals, working in a variety of contexts, than their acutely-ill counterparts. 


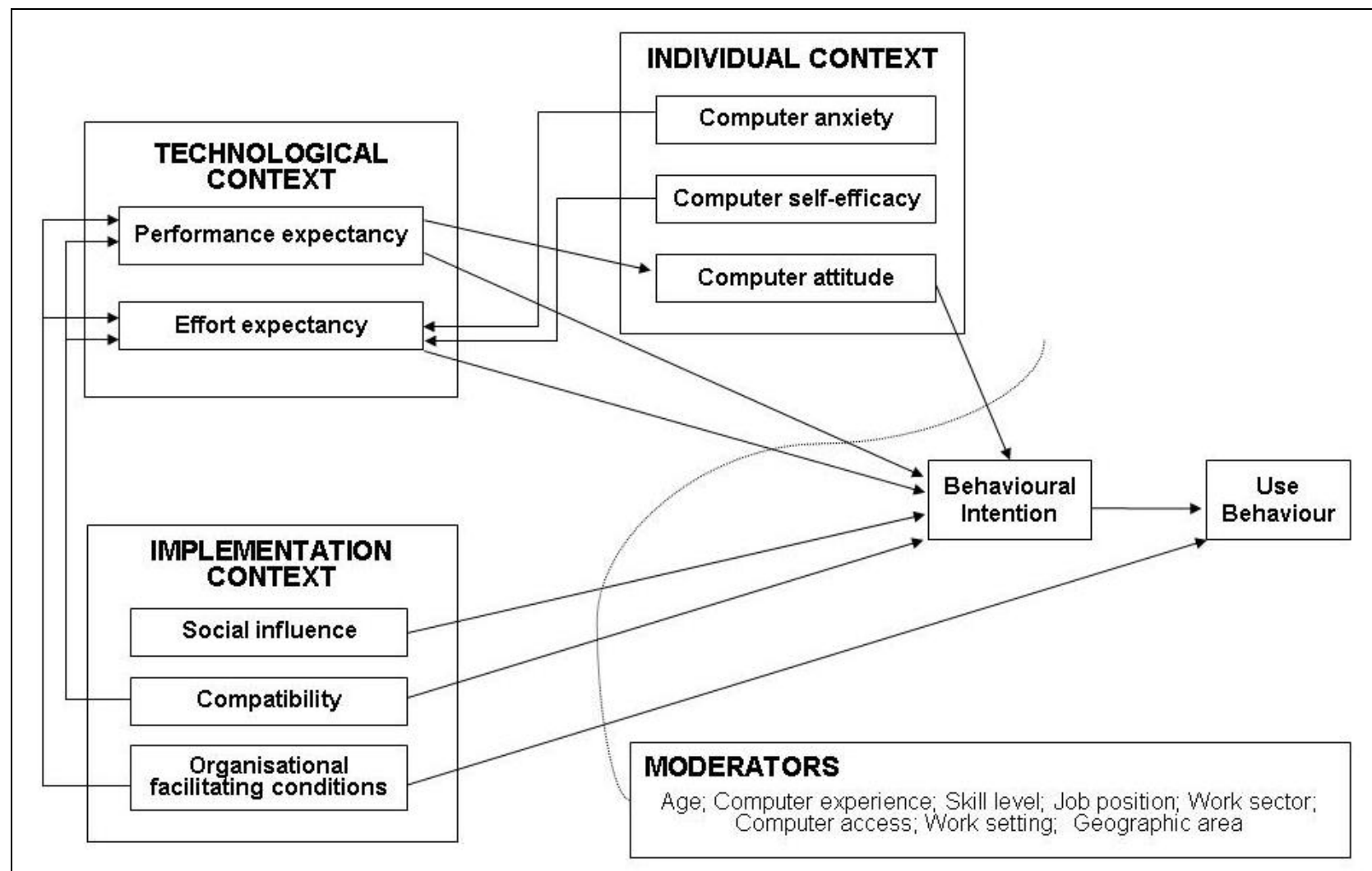

Figure 1. Research Model

The research reported in this paper attempts to address these issues by studying technology acceptance and use by Australian allied health therapists, particularly occupational therapists. Occupational therapists (OTs) are allied health professionals who provide support to a wide range of people with physical, psychological or developmental injuries or disabilities. They work in a wide variety of settings including private practice, hospitals, nursing homes, community centres and private industry and work with clients across the lifespan, from infancy to old age [27].

This paper provides a brief overview of the theoretical background and development of a model of technology acceptance and associated hypotheses. The methodology employed to empirically validate the model and to analyse the data is explained. The findings from the three-phase study are presented. The paper concludes with a discussion of the significance and implications of the results.

\section{Research Model and Hypotheses}

The research model developed in this study (Figure 1) draws on findings from relevant prior research and is primarily based on the UTAUT model [28] and on the generic framework for technology acceptance proposed by Chau \& Hu [29]. For a full discussion of the development of the research model and associated hypotheses see [30] and [31].

The Unified Theory of Acceptance and Use of Technology (UTAUT) was formulated by leading technology acceptance researchers and published in the September 2003 edition of MIS Quarterly [28]. The model was formulated based on conceptual and empirical similarities across 8 prominent competing technology acceptance models. UTAUT was empirically validated amongst 4 businesses in various industries (the health sector was a notable exception) and cross-validated using data from another 2. UTAUT was able to explain $70 \%$ of technology acceptance behaviour [28]. The UTAUT model was adapted for this research due to the comprehensiveness and rigour applied in its development and its high explanatory power.

Chau \& Hu's [29] 'three-dimensions framework' was adapted for this research due to its applicability to acceptance within the health sector and for its provision of contexts which assist a systematic 
examination of technology acceptance that can be targeted for recommendations to various stakeholder groups.

The research model theorises that technology acceptance has three dimensions: 1) characteristics of the individual; 2) characteristics of the technology; and, 3) characteristics of the implementation context. The characteristics of individual users are grouped within the individual context. The technological context refers to the characteristics of the technology itself and is made up of two determinants performance expectancy and effort expectancy. The implementation context refers to the specific professional environment of the user and includes the determinants of social influence, organisational facilitating conditions and compatibility. It is the implementation context which is theorised to have the predominant influence on user acceptance, as it is within this dimension that organisational and social issues are examined and, importantly, the compatibility of the technology with the clinical priorities of delivering positive outcomes for clients.

In this research model, compatibility is defined as the degree to which an innovation is perceived as being consistent with the existing practices, values, needs and experiences of the health care professional (adapted from [29, 32-34]). In a health care context, compatibility of a technology with the work practices, values, needs and experiences of the user becomes a crucial determinant in acceptance decision-making. The high value placed on the therapeutic relationship between a client and therapist can not be underestimated as it is a crucial tool in the therapeutic process. An innovation which is perceived to be incompatible with this process or incompatible with the ultimate aim of improved patient outcome, will ultimately lead to rejection of the innovation by health care professionals. This is emphasised by research conducted into telepsychiatry innovation in clinical practice [35]. In this instance, health care professionals who were initially championing the virtues of telepsychiatry, eventually found that the technology threatened deeply embedded professional constructs about the nature and practice of therapeutic relationships and was eventually rejected and resisted in a determined way [35].

The model is assumed to be influenced by variables such as age, IT experience, IT skill level, job position, work sector, work setting and geographical area - all of which are important characteristics which define Australia's OT population.

\subsection{Hypotheses}

Based on a review of the literature, the following hypotheses were developed in association with the research model:

H1: Computer attitude will positively affect the intensity of occupational therapists' behavioural intention.

H2: Computer self-efficacy will have a direct effect on effort expectancy.

H3: Computer anxiety will have a direct effect on effort expectancy.

H4: Computer self-efficacy will not have a significant effect on occupational therapists' behavioural intention.

H5: Computer anxiety will not have a significant effect on occupational therapists' behavioural intention.

H6: Performance expectancy will positively affect occupational therapists' attitude toward accepting technology.

H7: Performance expectancy will positively affect the intensity of occupational therapists' behavioural intention.

H8: Effort expectancy will positively affect the intensity of occupational therapists' behavioural intention.

H9: Social influence will positively affect the intensity of occupational therapists' behavioural intention.

H10: Organisational facilitating conditions will have a direct effect on effort expectancy.

H11: Organisational facilitating conditions will have a direct effect on performance expectancy.

H12: Organisational facilitating conditions will not have a significant influence on occupational therapists' behavioural intention.

H13: Organisational facilitating conditions will positively affect the intensity of occupational therapists' use behaviour.

H14: Compatibility will positively affect the intensity of occupational therapists' behavioural intention.

H15: Compatibility will have a direct effect on performance expectancy.

H16: Compatibility will have a direct effect on effort expectancy.

H17: Behavioural intention will have a significant positive influence on occupational therapists' use behaviour. 


\section{Methodology}

The proposed model was tested through a threephase study design, using mixed-mode methodology to collect substantial quantitative, qualitative and longitudinal data on technology acceptance and use amongst occupational therapists across Australia. Phase I consisted of the development of the preliminary research model, questionnaire development and pre-testing and conducting focus groups to obtain qualitative data. Phase II collected substantial survey data and phase III involved a longitudinal analysis of technology acceptance. The results of phase II and III are reported in this paper.

\subsection{Phase II - Survey}

Phase II involved a national survey sent to 6453 Australian occupational therapists to provide crosssectional data on behavioural intention and acceptance of ICT and other issues surrounding utilisation of ICT. For the purpose of the questionnaire, ICT was defined as a generalised term used to describe technologies that process information and aid communication. These technologies include, but are not limited to, a personal computer, various software programs, handheld computers, laptop computers, email and the Internet. Questionnaire content included 35 items designed to measure the constructs and relationships contained in the research model. Where possible, previously developed and validated items were used. Items were measured using a 7-point Likert-type scale, with $1=$ strongly disagree and $7=$ strongly agree. Questions were randomly arranged to reduce the potential ceiling or floor effect that may induce monotonous responses from participants. The strong theoretical underpinnings of the research model and the questionnaire items used to measure model constructs, strengthens instrument development by permitting the use of pre-specified and identified constructs [36].

\subsection{Phase III - Case Study}

A longitudinal multi-method field study was designed to examine ICT acceptance and use and the associated individual and organisational impacts of use or non-use within a small non-profit, communitybased health care organisation. The organisation (referred to as $\mathrm{KaK}$ ) provides specialised therapy and education services for children and families. It is predominantly dependent on Government funding. Of the 16 paid staff members only 5 are full time employees.
In $2004 \mathrm{KaK}$ was awarded an IS Grant to fund a complete overhaul of the existing IS. The pre-grant infrastructure at $\mathrm{KaK}$ was vastly inadequate and did not meet the organisation's needs. The IS implemented at KaK refers to the entire system, as changes were made to every aspect of the existing infrastructure. This included substantial changes to hardware, software, data and networks. KaK underwent a major undertaking, installing all elements of the new system over a weekend. When staff returned to work they were given a 3-hour group training session and were faced with major changes to their IS infrastructure and related processes.

Data collection was longitudinal over a 7-month timeframe, timed in conjunction with the introduction and use of the new computer system. Methods of data collection included questionnaires, interviews with members of the organisation, participant observation, a review of documentation and the installation of Visual Timeanalyzer, a time-use program which tracks all computer activities automatically and analyses these graphically [37]. The final section of the questionnaire contained the same 35 items used in phase II to measure the model constructs. The questionnaire was administered at three points in time: 1 week post-training, three months post-implementation and 7 months postimplementation.

\section{Results - Phase II}

\subsection{Study Respondents}

Thirty two (32\%) percent of the 6453 questionnaires distributed were returned. Of the 2044 responses received, 439 had incomplete responses that pertained to the measurement model. This analysis pertains to the remaining 1605 complete responses.

The average age of respondents was 37 years and 95\% were female. Based on the gender and age distributions of the data, the sample appears highly representative of the occupational therapist population within Australia [38]. OTs in management or senior roles made up $32.6 \%$ of the respondents and $13.7 \%$ were self-employed. The number of years of computer/ICT experience ranged from 1-30 years, with an average of 11.7 years. The majority $(66.5 \%)$ of respondents rated their computer skills as 'medium' and almost a quarter of the respondents believed they have low levels of computer skills. 


\subsection{Assessment of Measurement Model}

The partial least squares (PLS) approach of structural equation modelling (SEM) was used in this test of the research model. The PLS approach is most suited to this research as it supports both confirmatory and exploratory research. With its emphasis on identifying the variance and relationships between constructs and/or variables PLS permits exploration of the data and is well suited to accommodate complex theoretical and measurement models $[39,40]$. In addition, PLS does not require multivariate normality, permits models to be estimated using ordinal data derived from scales and is able to accommodate small sample sizes (of relevance to the breakdown of data in the subsequent analysis) $[39,40]$. The PLS technique has been used by IS researchers and those investigating technology acceptance, with particular note, Venketesh et al.'s development of the UTAUT model, on which this research model is based [28].

The analysis of the research model was conducted using PLS Graph v03.00 [41]. Model estimation involved two stages: firstly, the reliability and validity of the measurement model was assessed; and secondly the structural model was assessed. This insures that measures of the constructs are reliable and valid before assessing the relationships among the constructs [40]

To ensure the research model has acceptable measurement properties, reliability, internal consistency and discriminant validity were assessed. Individual item reliability was tested by using PLS to produce individual item loadings. Of the 35 items SI4, SE1, SE3 and SE4 had loadings of less than the acceptable level of 0.3 [42] and were removed from further analyses. As a result, computer self-efficacy (SE) has only the one item included in the analysis of the model. While this is statistically correct, using only one item to measure a construct limits the model's ability to adequately capture the entire concept and raises the potential for respondent error. It is recommended that future studies revise the indicators for SE.

The revised model with 31 indicators was again tested using PLS. All individual item reliabilities were above 0.662 and thus deemed reliable. All internal composite reliabilities were greater than the acceptable level of 0.70 [43]. The square roots of the shared variance between the constructs and their measures (AVE) were higher than the correlation of latent variables, with the exception of 2 values (COM/PE and COM/ATT). Thus while convergent and discriminant validity is good, it is not fully supported. These figures are displayed in Table 1.

\begin{tabular}{|l|c|c||c|c|c|c|c|c|c|c|c|}
\hline \multicolumn{10}{|c|}{ Table 1: Internal consistency, correlation of latent variables and square roots of AVE } \\
\hline & $\mathbf{N}^{\mathbf{0}}$ Items & ICR & PE & EE & ANX & SE & ATT & SI & COM & OFC & BI \\
\hline PE & 4 & 0.914 & $\mathbf{0 . 8 5 3}$ & & & & & & & & \\
\hline EE & 4 & 0.923 & 0.564 & $\mathbf{0 . 8 6 6}$ & & & & & & & \\
\hline ANX & 4 & 0.871 & -0.462 & -0.699 & $\mathbf{0 . 7 9 3}$ & & & & & & \\
\hline SE & 1 & 1.000 & 0.425 & 0.557 & -0.438 & $\mathbf{1 . 0 0 0}$ & & & & & \\
\hline ATT & 4 & 0.866 & 0.747 & 0.638 & -0.524 & 0.372 & $\mathbf{0 . 7 8 6}$ & & & & \\
\hline SI & 3 & 0.819 & 0.347 & 0.195 & -0.105 & 0.163 & 0.292 & $\mathbf{0 . 7 7 6}$ & & & \\
\hline COM & 4 & 0.819 & 0.800 & 0.541 & -0.484 & 0.411 & 0.730 & 0.355 & $\mathbf{0 . 7 2 9}$ & & \\
\hline OFC & 4 & 0.873 & 0.417 & 0.551 & -0.400 & 0.343 & 0.380 & 0.493 & 0.404 & $\mathbf{0 . 7 9 6}$ & \\
\hline BI & 3 & 0.871 & 0.729 & 0.578 & -0.476 & 0.422 & 0.711 & 0.351 & 0.719 & 0.400 & $\mathbf{0 . 8 3 2}$ \\
\hline \multicolumn{7}{|c|}{ Notes: } \\
1. The bold elements on the main diagonal are the square roots of AVE \\
2. PE: Performance expectancy; EE: Effort expectancy; ANX: Computer anxiety; SE: Computer \\
Self-efficacy; ATT: Computer attitude; SI: Social influence; COM: Compatibility; OFC: \\
Organisational facilitating conditions; BI: Behavioural intention \\
3. ICR: Internal composite reliability
\end{tabular}

\subsection{Assessment of Structural Model and Hypothesis Testing}

Table 2 shows model test results with direct effects only (ie the influence of moderating variables are not included in this analysis). The results of the PLS analysis with bootstrapping reveal that all of the 12 direct path hypotheses are statistically significant. The direct-effects only model explains $63 \%$ of the variance in behavioural intention. 
Table 2. Significance of Individual Direct Paths

\begin{tabular}{|c|c|c|c|}
\hline Path & $\begin{array}{c}\text { Path } \\
\text { coefficient }\end{array}$ & $\begin{array}{c}\boldsymbol{t} \text {-value for } \\
\text { path }\end{array}$ & Hypothesis \\
\hline $\mathrm{COM} \rightarrow \mathrm{PE}$ & 0.755 & $59.7216^{* * * *}$ & H15 \\
\hline $\mathrm{PE} \rightarrow \mathrm{ATT}$ & 0.747 & $58.7182 * * * *$ & H6 \\
\hline $\mathrm{ANX} \rightarrow \mathrm{EE}$ & -0.435 & $21.7849 * * * *$ & H3 \\
\hline $\mathrm{OFC} \rightarrow \mathrm{EE}$ & 0.243 & $12.3296^{* * * *}$ & H10 \\
\hline $\mathrm{SE} \rightarrow \mathrm{EE}$ & 0.226 & $11.5194 * * * *$ & H2 \\
\hline $\mathrm{PE} \rightarrow \mathrm{BI}$ & 0.258 & $7.8554 * * * *$ & H7 \\
\hline $\mathrm{ATT} \rightarrow \mathrm{BI}$ & 0.231 & $7.7468 * * * *$ & H1 \\
\hline $\mathrm{COM} \rightarrow \mathrm{BI}$ & 0.240 & $7.3779 * * * *$ & H14 \\
\hline $\mathrm{COM} \rightarrow \mathrm{EE}$ & 0.140 & $6.7638 * * * *$ & H16 \\
\hline $\mathrm{OFC} \rightarrow \mathrm{PE}$ & 0.112 & $6.6169^{* * * *}$ & H11 \\
\hline $\mathrm{EE} \rightarrow \mathrm{BI}$ & 0.140 & $5.9503 * * * *$ & H8 \\
\hline $\mathrm{SI} \rightarrow \mathrm{BI}$ & 0.082 & $4.4865 * * * *$ & H9 \\
\hline
\end{tabular}

Notes

1. Direct relationships only have been tested; figures do not account for indirect relationships

2. Figures were calculated by performing a bootstrapping resampling technique within PLS Graph, which uses randomly selected subsamples to generate t-statistics to indicate significance of model paths.

3. $\mathbf{R}^{\mathbf{2}}=\mathbf{0 . 6 3 4}$ (PLS, Direct effects-only model)

4. ****p-value $<0.0001$

While not discounting the relevance and importance of these statistical significance figures, the high statistical power is to be expected due to the large number of cases $(n=1605)$ which will produce small standard errors and make the statistical tests overly sensitive [44]. Measures of practical significance can be applied to this data to provide a further level of analysis to assess the substantive and theoretical implications of the relationships between the variables [44].

The path coefficients for $\mathrm{COM} \rightarrow \mathrm{PE}$ and $\mathrm{PE} \rightarrow$ ATT are both at 0.75 . Thus for every unit change in $\mathrm{COM}$ there is almost a one unit change in PE; the same applies to the effects of changes in PE on ATT. The relationship between these variable is thus more substantive than the relationship between OFC $\rightarrow \mathrm{PE}$, in which 9 unit changes in OFC would need to occur before there is a 1 unit change in PE.

So while tests of statistical significance show that all 12 direct paths are significant, tests of practical significance demonstrate that PE, ATT and COM have a more substantive influence on behavioural intention (BI) than does EE or SI.

To test the influence of moderating variables, PLS was used to generate path coefficients for each separate data set based on age, job position, computer experience and computer skills. The $\mathrm{R} 2$ values and hence the explanatory power of the model did not change significantly in each subset (ranged from $56.9 \%-67.8 \%$ ), despite significant changes in $n$ and the existence of differing moderating variables. This consistency across subpopulations is a sign of representative reliability and implies the model is robust as the $\mathrm{R} 2$ is high across subpopulations.

\subsection{Selected Descriptive Results}

The majority of respondents had access to a personal computer $(95 \%)$ and the Internet $(86 \%)$. Only 35\% used a laptop and 6\% reported using PDAs. A variety of clinical, administration \& professional uses of ICT were identified predominantly use of ICT for continuing professional development $(80 \%)$ and researching on the Internet (84\%).

An interesting and unexpected result of this phase of the research was the interest and support of this research by the occupational therapy community. Due to the length of the survey as well as the fact that this was the first time such research had been conducted, the researchers were optimistically hoping to receive $\sim 500$ survey responses. The actual response of 2044 exceeded expectations and was itself an indicator of the importance of this topic to the OT community. People contacted us requesting a copy of the survey and one person even asked for another copy because the snails in her mailbox had eaten it! In addition, the responses contained an excess of qualitative comments (more than space was allocated for), further demonstrating OTs interest in the topic of ICT acceptance and use. The majority of OTs expressed a high interest in the topic of technology and were interested to know more about how other health professionals and other OTs are incorporating ICT into their work. Further evidence of OT's positive attitudes and motivation levels towards ICT were that $89 \%$ of respondents want to increase their ICT skills/competency; $85 \%$ foresee an increase in the use of ICT in the next 5 years and $84 \%$ believe ICT can add positive value to their work as therapists.

Problems of access to ICT and the provision of training were cited and many therapists complained that their workplace did not give them adequate access to ICT. Many shared stories of how their one computer is "so old" and is shared by up to 10 other health professionals. 


\section{Results - Phase III}

The following information and discussion is provided which provides support for the research model and which demonstrates the unique influences upon allied health professionals' acceptance and use of ICT.

Of the 16 individuals employed at KaK, 14 consented to take part in this research. The following reports an analysis of responses from 9 key staff, 8 of whom were at $\mathrm{KaK}$ for the entire duration of the research and 1 of whom started 5 weeks after implementation of the new system. Participants included occupational therapists, physiotherapists, speech therapists, a psychologist, a therapy assistant and administration/management staff.

\subsection{Technology Use}

The use of ICT at KaK increased dramatically over the 7-month duration of this study. All participants reported an increase in frequency and extensiveness of use. While management staff reported a $60-250 \%$ increase in their use of computers, therapists reported a $100-150 \%$ increase.

Participants used computers for a variety of clinical, administration and professional purposes. Since implementing the new system: $67 \%$ of participants reported an increased use of computers during client interventions (i.e. as a tool in therapy); $78 \%$ increased their use of ICT to communicate with clients and/or families; $67 \%$ increased their use of computers to document client information and to schedule client appointments; and 56\% increased their use of ICT for continuing professional development and education. 100\% of participants foresee an increase in the use of ICT in their work in the next 5 years and $88 \%$ believe ICT can add positive value to their work.

All staff learnt new ways of using computers in their work. Examples observed by the researcher and reported by participants include: i) Clinical purposes, e.g. including digital photos and scanned images of a child's handwriting into a report that is saved in PDF format and emailed to the relevant external agencies; ii) Administrative purposes, e.g. utilising a laptop and data projector for staff meetings; and, iii) Therapeutic purposes, e.g. creating a PowerPoint presentation for a child as a motivating tool to improve hand-eye coordination and communication.

\subsection{Technology Acceptance}

Due to the small number of cases, statistical significance of the direct paths and moderating variables was unable to be determined. However, raw survey data and qualitative data collected during the course of the research lends support to the following constructs in the research model:

- Attitude - raw data from the questionnaire (T1 average $($ ave $)=6.09$ ), in addition to observation and interviews, illustrates all participants to have a positive attitude towards the new system. The positive attitude of staff surprised one interviewee who stated "(I'm) surprised staff have taken to it as well as they have. They've been really positive and taken it on board".

- Anxiety - participants reported varying levels of anxiety towards using the system and was not observed or reported ( $\mathrm{T} 1$ ave $=2.88$ ) to directly influence system use.

- Self-efficacy - The raw data (T1 ave = 4.47) indicates low-medium levels of computer selfefficacy for the majority of participants. This appeared to have little impact on use.

- Performance expectancy - A commonly cited benefit of the new system by staff is improvements in work productivity and efficiency $(n=8,88.8 \%)$. This was also reflected in the raw data on performance expectancy $(\mathrm{T} 1$ ave $=5.84)$.

- Effort expectancy - $(\mathrm{T} 1$ ave = 5.09). There was much variation within the organisation as to the preimplementation level of ICT knowledge and skills. Some staff quickly became skilful while others continue to experience difficulty. However, this variation does not appear to effect use of the system and it is possible that the effort required to learn and use the system is not a reliable indication of use.

- Social influence - although not receiving a high raw score (T1 ave =4.97), "peer pressure...in a good way", was cited by an interviewee as a factor contributing to ICT use at KaK. Many interviewees also referenced the ability to ask other staff for assistance as a factor contributing to their use of the system.

- Compatibility - the research model hypothesises that compatibility, the degree to which an innovation is perceived as being consistent with the existing practices, values, needs and experiences of the health care professional, is a key variable influencing acceptance and use. In support of this, high scores were attributed to the raw data $(\mathrm{T} 1$ ave $=6.22)$. Qualitative data suggests that a key specific feature of the IS at KaK that impacted system use and outcomes are its ability to meet individual and organisational needs and to enhance staff ability to carry out their core functions and maintain the goals and objectives of service delivery. 
- Organisational facilitating conditions - All interviewees attested to the support given to them by the management team. There was a unanimous belief that management "did their best", were supportive and encouraging and made themselves available to assist staff and answer their questions. Again, this is reflected in the high raw data score (T1 ave $=5.78)$. Conversely, the provision of training, resources and time to gain the knowledge and skills necessary to use the new system is also a part of this construct. It was widely acknowledged by all interviewees that the training provided to staff was inadequate and that provisions should have been made for allocating staff time to learn the new system and to transfer their data. However, it is possible that these potential barriers to acceptance and use did not pose barriers due to other contributing factors, as outlined below.

Analysis of the richness of the qualitative data collected throughout the research period has revealed additional factors which contributed to acceptance and use of the new system.

- Gender - KaK is a female dominated organisation. With the exception of one volunteer (Ian) and a parttime psychologist (who was not available to take part in this study), all employees are female. While gender was not a focus of this study, it is hypothesised to be a moderating variable in the research model. It is important to note that alliedhealth organisations are often female dominated and this is an important factor to investigate in future acceptance research in the health sector.

- IT support - this is provided by a volunteer whose role has extended to a virtually full-time position. He is constantly on-hand to answer staff questions and to trouble-shoot technical problems. All staff have grown reliant on his presence and the CEO attributed the success of IS implementation to him. This attests as to the importance of IT support in contributing to use and acceptance.

- Computer access - access is a moderating variable in the research model. Although unable to be quantitatively measured, the majority of staff cited the ability to access a computer when needed as a key feature of their increased use of ICT.

- Funding - as a non-profit organisation, predominantly reliant on Government funding, all staff are acutely aware of the constant financial and resource constraints. As a result, staff were appreciative of their "windfall" and were excited about "entering the Information Age"; as one staff member stated "if we don't have this, we don't have anything". This added to staff motivation to use the new system.
- Other individual characteristics. All participants were asked to describe why they and others persisted in their use of the system when faced with continual technical problems and other barriers. The responses were concisely captured by one who stated "Bloodymindedness!...We are all pretty persistent, dogged and determined. We've gone this far and we've made the commitment, and we (know) we can do it". These individual characteristics of persistence and determination may be worth including in future technology acceptance studies.

- Altruism as part of the organisational culture. The structure and culture of an organisation may influence acceptance, use and IS success. This has been particularly evident at $\mathrm{KaK}$. Although the organisational chart shows some hierarchy in the organisational structure, the organisation fosters a unique sense of community amongst staff and volunteers. They work as a team and often provide services as part of a multi-disciplinary team. All staff contribute to the decision-making process and can be involved as much, or as little as they choose, in the operations of the organisation. Acceptance and use of the new system was viewed by staff as an opportunity to enhance the service provided to $\mathrm{KaK}$ clients and their families and their motivation highlights staff commitment to the organisation, its missions and goals.

Also important were the links between technology acceptance and use and the matching of organisational and individual goals. The individual goals of system implementation and use were closely aligned to the organisational goals. Although this did not appear deliberate, it further attests to the organisational culture, commitment and values of the organisation and the individuals it employs. The alignment of the goals fostered a sense of "we're all in this together" and ensured everyone aimed towards similar outcomes which added to individual motivation to use the system.

\subsection{Success of the IS implementation}

The IS implementation at KaK was deemed a success by study participants, as indicated by: improved access, increased productivity, decreased stress, improved time management/efficiency, and staff acceptance and use. The success of this implementation has continued past the research period and the organisation and the therapists have "gone from strength to strength" in their use of ICT. Acceptance of the technology translated into use of the technology; which evolved into dependence on technology and a commitment to increase their use of ICT wherever possible. ICT is now seen as an 
essential tool and staff have become reliant on its use. IS failures have resulted in staff dissatisfaction and claims being made that they "can't do their work".

This success was despite a number of significant challenges which occurred post-implementation, including: the fast pace of change; inadequate training provided to staff; no time given to staff to support their adjustment to and learning of the new system; and underdevelopment of policies, procedures and guidelines for use. They were also plagued with technical problems which include a longer-than expected implementation period; an inadequate infrastructure; incorrect configuration of the network and consistent error messages and system crashes.

\section{Conclusion}

This paper presents an overview of the development and testing of a model of technology acceptance by Australian occupational therapists. Results from this study demonstrate both quantitative and qualitative support for the research model. The analysis performed to date and presented in this paper demonstrates that all 12 direct path hypotheses are statistically significant, and that compatibility (COM), attitude (ATT) and self-efficacy (SE) exhibit a higher practical significance on intention to use ICT than effort expectancy (EE) or social influence (SI). The direct-effects only model explained $63 \%$ of the variance in behavioural intention to use ICT. The preliminary analysis of the influence of age, job position, computer experience and computer skills as potential moderating variables provides further evidence of the robustness of the model and demonstrates the value of including moderating factors to help explain and describe the phenomenon of technology acceptance.

Results from the case study demonstrate qualitative support for the research model and provide rich information on other factors that may influence allied health therapists' ICT acceptance and use decisions. Factors such as altruism, individual commitment to the organisation and motivation were identified as contributing to the acceptance and use of the new system.

This work has highlighted the complexity of the constructs and relationships that influence technology acceptance and successful implementation outcomes. Much of this complexity is not addressed in many of the technology acceptance models, which often aim for simplistic description of the factors at play and hence are limited in their ability to understand and describe this complexity. It is suggested that these factors be considered in future developments and extensions of technology acceptance models and research in the health sector.

Information from the case study and the survey support the position that OT's are highly motivated and hold positive attitudes towards ICT and the potential benefits of technology use for them and the clients they serve. Results show that while the factors influencing individual technology acceptance and use decisions by healthcare professionals vary from those factors that apply to business professionals and students; that there may be some particularly unique factors that contribute to allied health therapists and those working in community based settings. Further broadening of enquiries of technology acceptance to include non-medical health professionals will only aid our understanding of the various complexities of IS implementation in the health sector and will generate knowledge which can be applied to improve the success of information system implementation in this arena.

\section{Acknowledgements}

The authors would like to sincerely thank Kids Are Kids and Australia's occupational therapy community for their interest in and support of this research.

\section{References}

[1] Schuring, R. and T. Spil. "Mini Track: Evaluation of implementation, adoption and diffusion of IS in Healthcare (HCADI)", in 37th Annual Hawaii International Conference on System Sciences (HICSS'04). 2004. Big Island, Hawaii: Computer Society Press.

[2] Heeks, R., "Health information systems: Failure, success and improvisation", International Journal of Medical Informatics, 2006, 75: pp. 125-137.

[3] Chismar, W.G. and S. Wiley-Patton. "Does the extended technology acceptance model apply to physicians", in 36th Hawaii International Congress on System Sciences (HICSS'03). 2003. Big Island, Hawaii: IEEE Computer Society.

[4] Dearne, K., "Health's tech bypass", in The Australian. 2003: Sydney. pp. 1, 4.

[5] Murray, D., "Healthcare challenge", in Australian Information Week. 2002. pp. 10-18.

[6] Remmlinger, E. and M. Grossman. "Physician utilization of information systems: bridging the gap between expectations and reality", in 1992 Annual Healthcare Information and Management Systems Society (HIMSS) Conference. 1992.

[7] Wenn, A., et al. "A sociotechnical investigation of factors affecting I.T. adoption by rural GPs", in Information Technology in Regional Areas. Using IT: Make IT Happen. 2002. Rockhampton, Qld Australia: Online.

[8] Western, M., et al., Measuring IT use in Australian General Practice, ed. G.P.C.G. (GPCG). 2001, Australia: University of Queensland. 
[9] "House Subcommittee Passes Amended Health IT Bill", in iHealthBeat. 2006: Californian Healthcare Foundation. [10] Hillestad, R., et al., "Can Electronic Medical Record Systems Transform Healthcare? Potential Health Benefits, Savings and Costs", Health Affairs, 2005, 24(5).

[11] Gagnon, M.-P., et al. "The impact of organizational characteristics on telehealth adoption by hospitals", in 37th Hawaii International Conference on System Sciences (HICSS'04). 2004. Big Island, Hawaii: IEEE Computer Society.

[12] Zheng, K., et al., "Understanding technology adoption in clinical care: Clinician adoption behaviour of a point-ofcare reminder system", International Journal of Medical Informatics, 2005, 74: pp. 535-543.

[13] Scott, J.T., et al., "Kaiser Permanente's experience of implementing an electronic medical record: a qualitative study", British Medical Journal, 2005, ONLINE: pp. 1-5. [14] Lua, Y.-C., et al., "A review and a framework of handheld computer adoption in healthcare", International Journal of Medical Informatics, 2005, 74: pp. 409-422. [15] Selder, A., "Physician reimbursement and technology adoption", Journal of Health Economics, 2005, 24(5): pp. 907-930.

[16] Dreiseitl, S. and M. Binder, "Do physicians value decision support? A look at the effect of decision support systems on physician opinion", Artificial Intelligence in Medicine, 2005, 33: pp. 25-30.

[17] Reece, R., "The four C's of physician EMR adoption". 2004, HealthLeaders.

[18] Miller, R. and I. Sim, "Physicians' Use of Electronic Medical Records; Barriers and Solutions", Health Affairs, 2004, 23(2): pp. 116-125.

[19] Lai, T.Y.Y., et al., "Do doctors act on their selfreported intention to computerize? A follow-up populationbased survey in Hong Kong", International Journal of Medical Informatics, 2004, 73: pp. 415-431.

[20] Horan, T.A., et al. "Use of online systems in clinical medical assessments: an analysis of physician acceptance of online disability evaluation systems", in 37th Hawaii International Conference on System Sciences (HICSS'04). 2004. Big Island, Hawaii: IEEE Computer Society. [21] Han, S., et al. "Physicians' behavior intentions regarding a mobile medical information system: An exploratory study", in The Tenth Americas Conference on Information Systems. 2004. New York, New York.

[22] Tulu, B., B. Hilton, and T.A. Horan. "Physicians' acceptance of web-based medical assessment systems: findings from a national survey", in 9th Americas Conference on Information Systems (AMCIS 2003). 2003. Tampa, Florida USA: Association for Information Systems. [23] Pomeroy, C., et al. "Physician adoption of an electronic clinical information system", in 2003 Annual HIMSS Conference and Exhibition. 2003. San Diego: Online.

[24] Yi, M.Y., et al., "Understanding information technology acceptance by individual professionals: Toward an integrative view", Information \& Management, 2006, 43: pp. 350-363.

[25] American Health Information Community, "Chronic Care Workgroup", in American Health Information Community Workgroups. 2006.
[26] "A Road Map for Health IT in Long Term Care". 2005, AHIMA, AAHSA/CAST, AHCA/NCAL, AMDA, NAHC, and NASL: Online. pp. 12.

[27] World Federation of Occupational Therapists, "What is occupational therapy?" 2003, WFOT.

[28] Venkatesh, V., et al., "User acceptance of information technology: toward a unified view", MIS Quarterly, 2003, 27(3): pp. 425-478.

[29] Chau, P.Y.K. and P.J.-H. Hu, "Examining a model of information technology acceptance by individual professionals: an exploratory study", Journal of Management Information Systems, 2002, 18(4): pp. 191229.

[30] Schaper, L.K. and G.P. Pervan, "ICTs and OTs: A model of information and communication technology acceptance and utilisation by occupational therapists", International Journal of Medical Informatics, 2006, in press.

[31] Schaper, L.K. and G.P. Pervan. "A model of information and communication technology acceptance and utilisation by occupational therapists", in 2004 IFIP International Conference on Decision Support Systems. 2004. Prato, Italy.

[32] Moore, G.C. and I. Benbasat, "Development of an instrument to measure the perception of adopting an information technology innovation", Information Systems Research, 1991, 2(3): pp. 192-223.

[33] Rogers, E.M., Diffusion of Innovations, 4th ed. 1995, New York: The Free Press.

[34] Taylor, S. and P.A. Todd, "Understanding information technology usage: a test of competing models", Information Systems Research, 1995, 6(4): pp. 144-176.

[35] May, C., et al., "Resisting and promoting new technologies in clinical practice: the case of telepsychiatry", Social Science and Medicine, 2001, 52(12): pp. 1889-1901. [36] Straub, D.W. and C.L. Carison, "Validating instruments in MIS research", MIS Quarterly, 1989, 13(2): pp. 147-169.

[37] Neuber, M., "Visual TimeAnalyzer". 2004, A. \& M. Neuber GbR,: Halle, Germany.

[38] AIHW, "Occupational Therapy Labour Force 1998". 1998, Australian Institute of Health and Welfare.

[39] Chin, W.W., "The partial least squares approach for structural equation modeling", in Modern Methods for Business Research, G.A. Marcoulides, Editor. 1998, Lawrence Erlbaum Associates: Hillsdale, NJ. pp. 295-336. [40] Barclay, D., R. Thompson, and C. Higgins, "The partial least squares (PLS) approach to causal modeling, personal computer adoption and use as an illustration", Technology Studies, 1995, 2(2): pp. 285-324.

[41] Chin, W.W. and T. Frye, "PLS Graph". 1996, Soft Modeling Inc: Calgary, Canada.

[42] Igbaria, M., et al., "Personal computing acceptance factors in small firms: a structural equation model", MIS Quarterly, 1997, 21(3): pp. 279-302.

[43] Nunnally, J., Psychometric theory, 2nd ed. 1978, New York: McGraw-Hill.

[44] Hair, J.F., et al., Multivariate Data Analysis, 5th ed. Vol. International Edition. 1998, New Jersey: Prentice-Hall Inc. 730 . 